\title{
Alopecia areata de placas múltiples. Comunicación de cinco casos tratados con PUVAsol
}

\author{
Multiple plate alopecia areata. Communication \\ of 5 cases treated with PUVAsol
}

\author{
María Antonieta Domínguez-Gómez, ${ }^{\star}$ Martha Alejandra Morales-Sánchez, ${ }^{\ddagger}$ \\ Paulina Rita Corona-Castillo, $\$$ Karla Noemí Salazar-Villegas, ${ }^{\S}$ César Alejandro Reyes-Salcedo§
}

\section{RESUMEN}

La alopecia areata es un trastorno autoinmune que se caracteriza clínicamente por la presencia de placas alopécicas de tamaño y número variable que adquieren una disposición circular, ubicadas generalmente en piel cabelluda y barba. Se le clasifica dentro del grupo de las alopecias no cicatrizales. Para iniciar un tratamiento adecuado se deben tomar en cuenta la extensión y las áreas anatómicas afectadas. Cuando la alteración es mayor de $50 \%$ en piel cabelluda y/o existen varias regiones involucradas, se indica el uso de fototerapia, más un psoraleno tópico $u$ oral. Algunos autores han observado resultados favorables con esta opción terapéutica para tratamiento de las alopecias totales y universales, otros no han encontrado beneficio alguno para la forma universal. Pacientes tratados con PUVAsol en la Unidad de Fototerapia del Centro Dermatológico «Dr. Ladislao de la Pascua» evolucionaron en forma favorable con repoblación de casi $100 \%$, por lo que se puede concluir que el empleo de la fototerapia para el manejo de la alopecia areata en placas múltiples y diseminadas puede ser una buena opción de tratamiento, la cual proporciona resultados adecuados. Sin embargo, se requiere realizar más ensayos clínicos aleatorizados para conocer el beneficio real de la PUVAterapia.

Palabras clave: Alopecia areata, PUVAterapia, psoraleno, repoblación.

\section{ABSTRACT}

Alopecia areata is as an autoimmune disorder, clinically characterized by hair loss in circular arrangement. It is classified into the non-scarring alopecias group. In order to initiate the correct treatment, it must be taken into account the extention and the anatomic areas affected at the time of diagnostic. When the disease affects greater than $50 \%$ of the scalp, and/or there are many areas involve, it is indicated to use phototherapy with topical or oral psoralen. Some authors have found positive results using this treatment in total and universal alopecia types, however, some others have found negative results in universal alopecia types. Patients treated with PUVAsol in the Phototherapy Unit of Centro Dermatológico "Dr. Ladislao de la Pascua", evolved favorably, with repopulation of almost $100 \%$, so it can be concluded that, phototherapy as treatment for multiple and disseminated alopecia areata plates, it is a good treatment option. Nevertheless, it is required to perform more randomized clinical trials to know the real benefit of the PUVA therapy.

Keywords: Alopecia areata, PUVA therapy, psoralen, repopulation.

\section{INTRODUCCIÓN}

La alopecia areata (AA) es una tricopatía autoinmune no cicatrizal. Su prevalencia en Europa se calcula que puede

\footnotetext{
* Jefa de la Clínica de Fototerapia.

‡ Jefa de Enseñanza e Investigación.

$\S$ Dermatólogo.
}

Centro Dermatológico «Dr. Ladislao de la Pascua», SSCDMX. llegar a 0.5-1 por 1,000 habitantes. ${ }^{1}$ En el Centro Dermatológico «Dr. Ladislao de la Pascua» representa 1.7\% del total de la consulta. ${ }^{2}$ En su etiopatogenia están implicados factores genéticos, autoinmunes y externos. Clínicamente se caracteriza por la presencia de placas alopécicas de

Citar como: Domínguez-Gómez MA, Morales-Sánchez MA, Corona-Castillo PR Salazar-Villegas KN, Reyes-Salcedo CA. Alopecia areata de placas múltiples. Comunicación de cinco casos tratados con PUVAsol. Rev Cent Dermatol Pascua. 2021; 30 (1): 5-10. https://dx.doi.org/10.35366/100585 
diferente tamaño y número, ubicadas principalmente en piel cabelluda y zona de la barba. El tratamiento depende de la edad del paciente, extensión de la dermatosis, tiempo de evolución, y estado de salud general. ${ }^{3}$

La (AA) es una enfermedad multifactorial, autoinmune y poligénica. Es una causa común de alopecia no cicatrizal. ${ }^{1}$

Se reporta en la literatura una frecuencia de $1-2 \%$ en la población en general. En la consulta dermatológica es de $1 \%$, sin predominio de edad y sexo. La incidencia es mayor entre la tercera y cuarta décadas de la vida, ${ }^{2}$ aunque en un alto porcentaje el primer episodio aparece entre los cinco y 20 años de edad. ${ }^{3,4}$

Representa la causa más frecuente de alopecia inflamatoria, con una patogenia aún no dilucidada. Se sugiere predisposición genética y alteraciones en el sistema inmunitario. Han sido implicados en la enfermedad los antígenos leucocitarios humanos (HLA) PTPN22 y AIRE así como los antígenos de histocompatibilidad HLA-DR5, relacionados con las formas graves; el HLA DR4 con las formas agudas, severas o ambas. Se han identificado 139 polimorfismos de nucleótidos ligados a la alopecia areata en ocho regiones del genoma asociados a la activación, proliferación y modulación de respuesta inmunitaria de células T. ${ }^{5}$

Clínicamente se clasifica de acuerdo a:

1. Su extensión: en placas, total o universal.

2. Patrón de caída: focal, multifocal, ofiásica, sisaifo, reticular, difusa, enrocada, tipo alopecia androgenética con patrón masculino o femenino o perinevoide. ${ }^{2}$

3. Patrones atípicos: lineal y perinevoide. ${ }^{1}$

Entre 1 y $2 \%$ de los casos afecta la totalidad de piel cabelluda.

Las formas leves suelen resolver en semanas o meses, las formas severas con pérdida de pelo completa o persistente se observan más frecuentemente en niños y adultos jóvenes o bien en adultos que cursan con comorbilidades autoinmunes. ${ }^{5}$

Datos de centros de referencia de segundo y tercer nivel indican que de 34 a $50 \%$ de los pacientes con los tipos leves se recuperan en un año, más de $50 \%$ experimentarán más de un episodio, y de los casos con alopecia total o universal menos de $10 \%$ se recuperará en forma total. ${ }^{4}$

El tratamiento de la AA es variable, por lo que el verdadero reto en esta patología es proporcionar un régimen de tratamiento adecuado. Al momento de elegir alguno de ellos se debe tomar en cuenta el impacto en la calidad de vida y la influencia que tienen los factores de mal pronóstico tales como: extensión, tiempo de evolución, edad y antecedentes familiares. Además, se deben conocer los efectos colaterales y complicaciones que pueden provocar los tratamientos a corto y largo plazo, e igualmente establecer un periodo de tres meses antes de hacer una valoración de los resultados.

Los medicamentos de primera línea más utilizados son los esteroides tópicos, la tretinoína y el minoxidil así como los esteroides intralesionales, aunque generalmente tienen un porcentaje de éxito limitado, particularmente en los casos extensos, por lo que se recomienda su empleo sólo cuando se calcula una afección menor de $50 \%$ en piel cabelluda, de preferencia se sugiere el uso de triamcinolona $5 \mathrm{mg} / \mathrm{mL}$ con vigilancia estrecha. En caso de no observarse mejoría después de seis meses de aplicación, se recomienda suspender el medicamento. Con este tratamiento $71 \%$ de los pacientes ha presentado mejoría, con una repoblación de 64 a 97\%. ${ }^{6}$

Cuando la afectación es mayor de $50 \%$ está indicado el uso de la inmunoterapia con difenilciclopropenona; sin embargo, tener acceso a este fármaco es difícil, por lo que se recomienda el uso de corticoides por vía oral en pulsos, o bien fototerapia. ${ }^{1}$

El empleo de la fototerapia fue sugerido por primera vez por Weismann y colaboradores como una alternativa terapéutica para la AA resistente a otros tratamientos al considerar su efecto inmunomodulador en el folículo piloso que actúa sobre los linfocitos T y la presentación de antígenos, inhibiendo el daño hacia la estructura capilar al reducir el número de células de Langerhans y las células del infiltrado inflamatorio. Desde entonces se han publicado varios estudios con respecto a su eficacia. ${ }^{6}$ Se han probado distintas modalidades para el uso de 8-metoxipsoraleno vía oral, tópica o en forma de turbante, $42 \%$ de los pacientes obtuvo buena respuesta con dicho tratamiento. ${ }^{7}$

Herz-Ruelas y colaboradores realizaron un estudio prospectivo para la evaluación de la dosis óptima, inicial y de incremento de UVA-1 en pacientes con AA sin mejoría al utilizar previamente los tratamientos de primera línea (esteroides tópicos, intralesionales, ácido retinoico, minoxidil o ciclosporina). Los 22 pacientes recibieron 75 sesiones de UVA-1 durante seis meses con un esquema de tres a cinco veces por semana a dosis de $30 \mathrm{~J} / \mathrm{cm}^{2}$. La dosis fue incrementada a $60 \mathrm{~J} / \mathrm{cm}^{2}$ y a $120 \mathrm{~J} / \mathrm{cm}^{2}$ en pacientes sin respuesta a tratamiento inicial. Se observó una mejoría más evidente con la dosis de $60 \mathrm{~J} / \mathrm{cm}^{2} .{ }^{8}$

Otro estudio realizado por Whitmot y colaboradores con 26 pacientes con alopecia total y/o universal tratados con 8-metoxipsoraleno oral o tópico y PUVA se obtuvo repo- 
blación por arriba de $90 \%$ en ocho enfermos con alopecia total, y en seis con universal, a los 7.4 y 10.4 meses en promedio respectivamente. ${ }^{4}$ En otro análisis realizado por Mohamed y colaboradores en 124 pacientes estudiados con varias formas clínicas de AA, y 25 con las formas total y universal, todos tratados con psoraleno tópico y PUVA, se encontró que en las dos últimas $56 \%$ mostraron repoblación de piel cabelluda y otras áreas pilosas después de tres ciclos. $84 \%$ de los casos mostró crecimiento por arriba de $50 \%$ después de uno a dos ciclos.

El uso de la fototerapia con régimen fototóxico de PUVA, (PUVAsol en los casos comunicados en este artículo), durante tres meses, representa una alternativa prometedora para el tratamiento de la AA total y universal. En estudios aleatorizados y controlados, $45 \%$ de los pacientes evolucionó en forma adecuada con este régimen. ${ }^{9}$

El uso de UVB de banda estrecha no ha demostrado ser eficaz en estos casos. Las dosis semanales de 200 $\mathrm{mJ} / \mathrm{cm}^{2}$ y $300 \mathrm{~mJ} / \mathrm{cm}^{2}$ para pacientes de fototipo II y III respectivamente fueron administradas, sin mostrar mejoría. ${ }^{10}$

\section{CASOS CLÍNICOS}

\section{Caso 1}

Escolar del sexo femenino de 12 años de edad, con placas alopécicas y pseudoalopécicas en piel cabelluda, cejas y pestañas, de cuatro meses de evolución, tratadas previamente con esteroide y retinoide tópico por seis meses, sin mejoría. Se inicia tratamiento con 8-metoxipsoraleno vía oral a dosis de $15 \mathrm{mg}$, con exposición a luz de sol a través de una ventana por 30 minutos, tres veces por semana (PUVAsol), presentando mejoría del cuadro a los 18 meses (Figuras 1 y 2).

\section{Caso 2}

Escolar del sexo masculino de 12 años de edad, con placas alopécicas en piel cabelluda, cejas y pestañas, de seis meses de evolución, tratado previamente con ácido acético glacial y esteroide tópico, sin mejoría. Se inicia con 8-metoxipsoraleno tópico y exposición a luz de sol a través de una ventana durante 30 minutos (PUVAsol) diario, presentando mejoría del cuadro a los 12 meses (Figuras 3 y 4).

\section{Caso 3}

Escolar del sexo femenino de 10 años de edad, con placas alopécicas en piel cabelluda, cejas, pestañas y

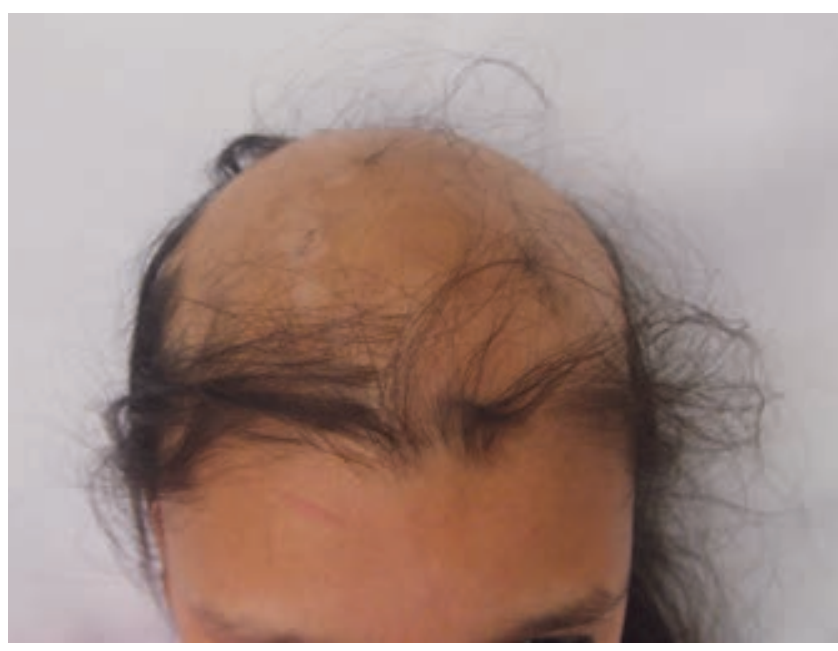

Figura 1: Placas alopécicas en piel cabelluda.

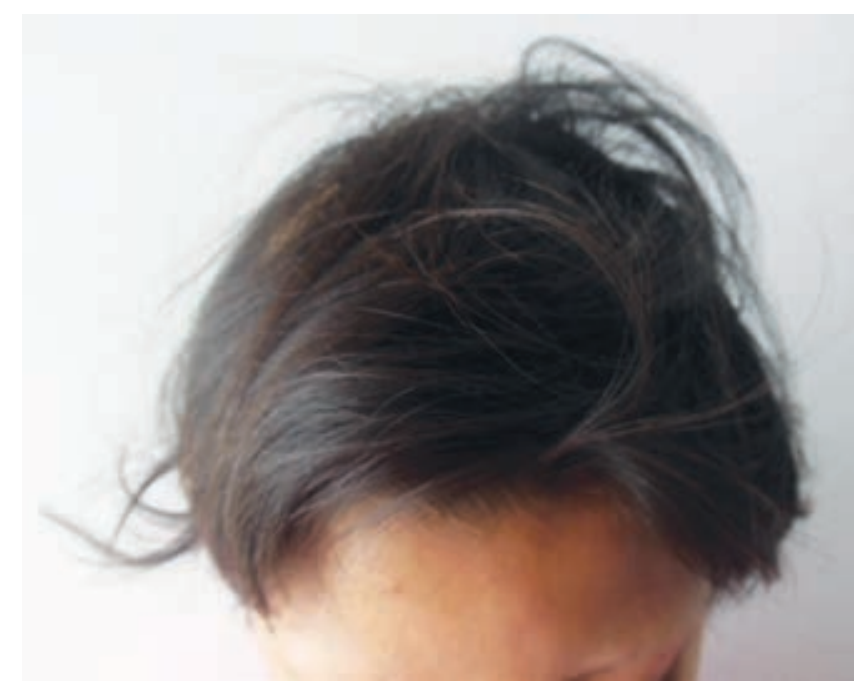

Figura 2: Mejoría de $70 \%$.

extremidades superiores, de cinco meses de evolución, tratada inicialmente con esteroide tópico por cuatro meses, sin mejoría. Se inicia fórmula magistral con esencia de lima al $50 \%$ diario por la noche, y al día siguiente exposición a luz de sol a través de una ventana por 30 minutos (PUVAsol), con mejoría completa a los 14 meses (Figuras 5 y 6).

\section{Caso 4}

Adolescente del sexo femenino de 15 años de edad, con placas alopécicas en piel cabelluda y pestañas de tres años de evolución, tratada previamente con 
esteroide tópico y minoxidil al 5\%, sin respuesta, y con múltiples recaídas. Se inicia tratamiento con psoraleno vía oral $20 \mathrm{mg}$ y exposición a luz de sol a través de una ventana por 30 minutos (PUVAsol) tres veces por semana, con repoblación completa a los 20 meses (Figuras 7 y 8 ).

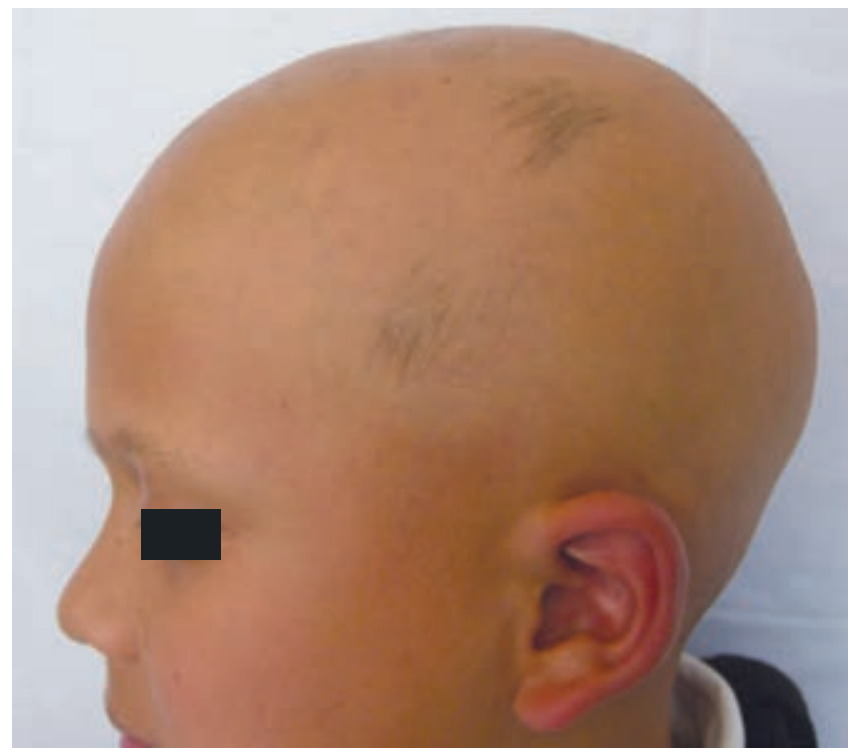

Figura 3: Placas alopécicas en piel cabelluda, cejas y pestañas.

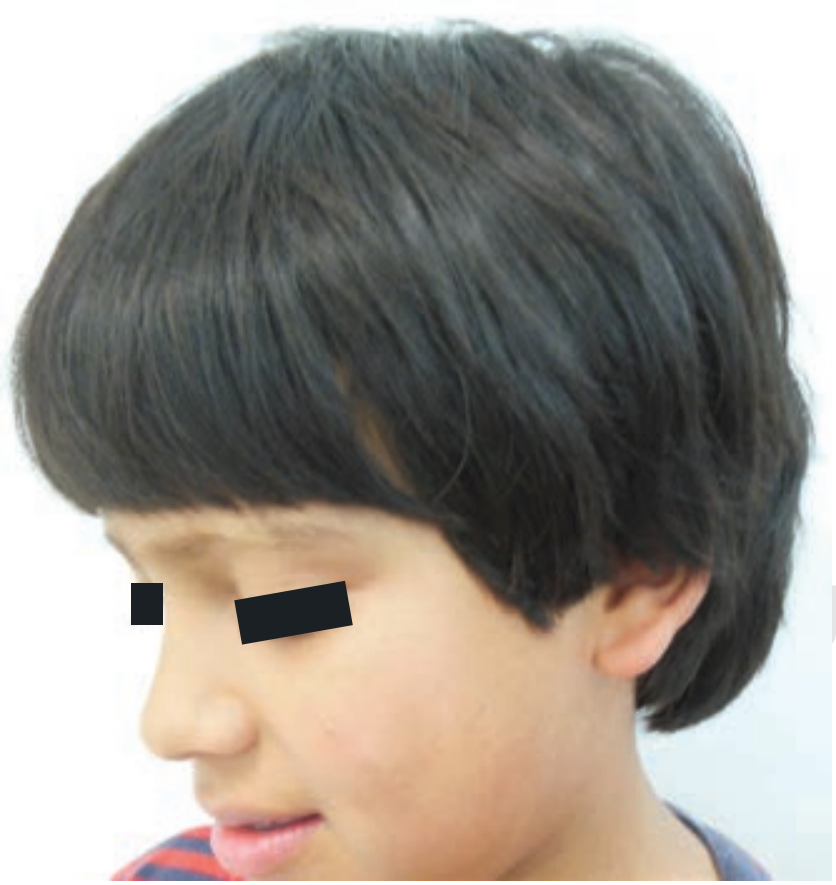

Figura 4: Repoblación de 90\%.

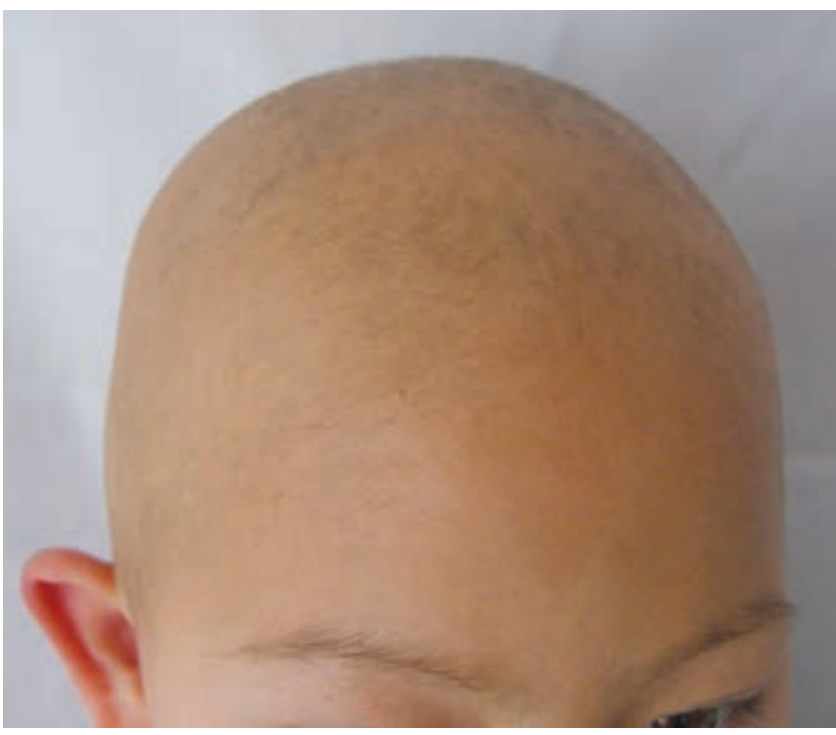

Figura 5: Placas alopécicas en piel cabelluda y cejas.

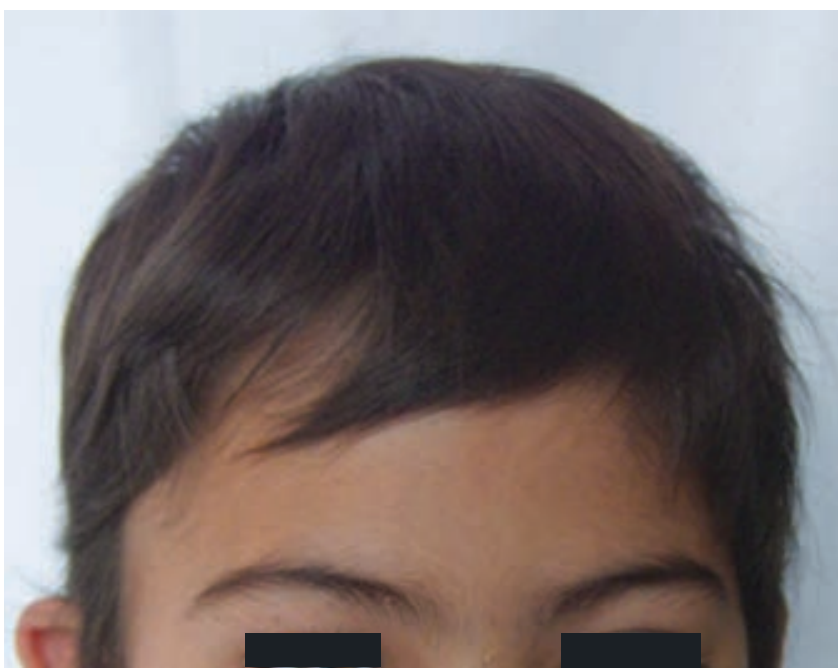

Figura 6: Repoblación completa de pelo.

\section{Caso 5}

Masculino de 20 años de edad, con placas alopécicas en piel cabelluda y cejas, de tres meses de evolución, tratado con esteroide tópico y minoxidil al 5\%, sin respuesta. Se inicia con 8-metoxipsoraleno ungüento y exposición a luz de sol a través de una ventana por 30 minutos (PUVAsol) diario, con repoblación de $70 \%$ a los 16 meses de tratamiento (Figuras 9 y 10). 


\section{COMENTARIO}

El curso natural de la AA es variable, presenta recaídas frecuentes, lo que hace complicado su tratamiento. La terapia PUVA ha demostrado ser eficaz en los casos en los que se ve afectado más de $50 \%$ de la piel cabelluda, o bien cuando se ven involucradas otras áreas pilosas.

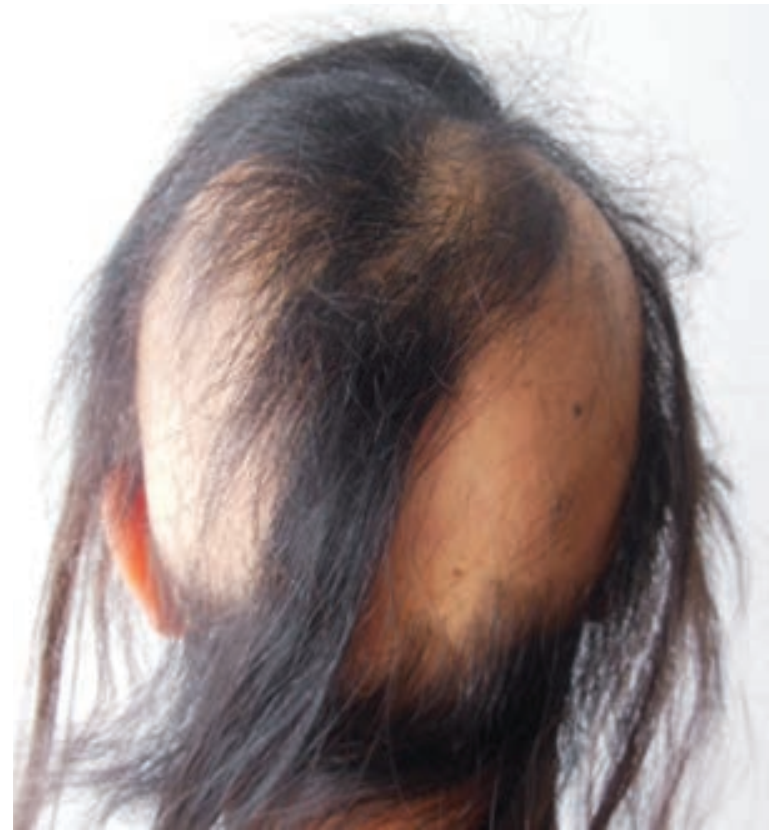

Figura 7: Placas alopécicas en piel cabelluda que abarcan grandes áreas.

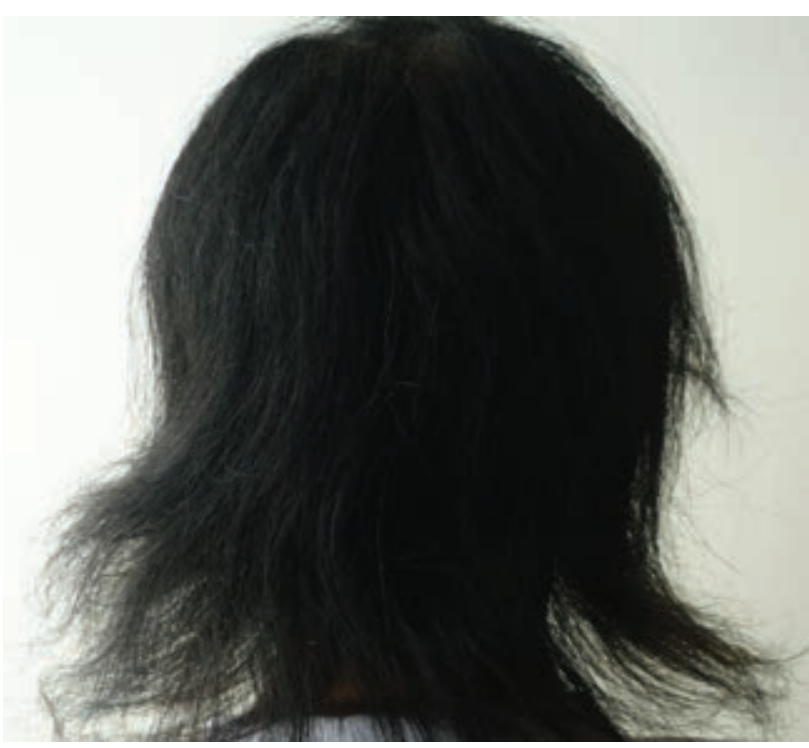

Figura 8: Crecimiento completo del pelo en piel cabelluda.

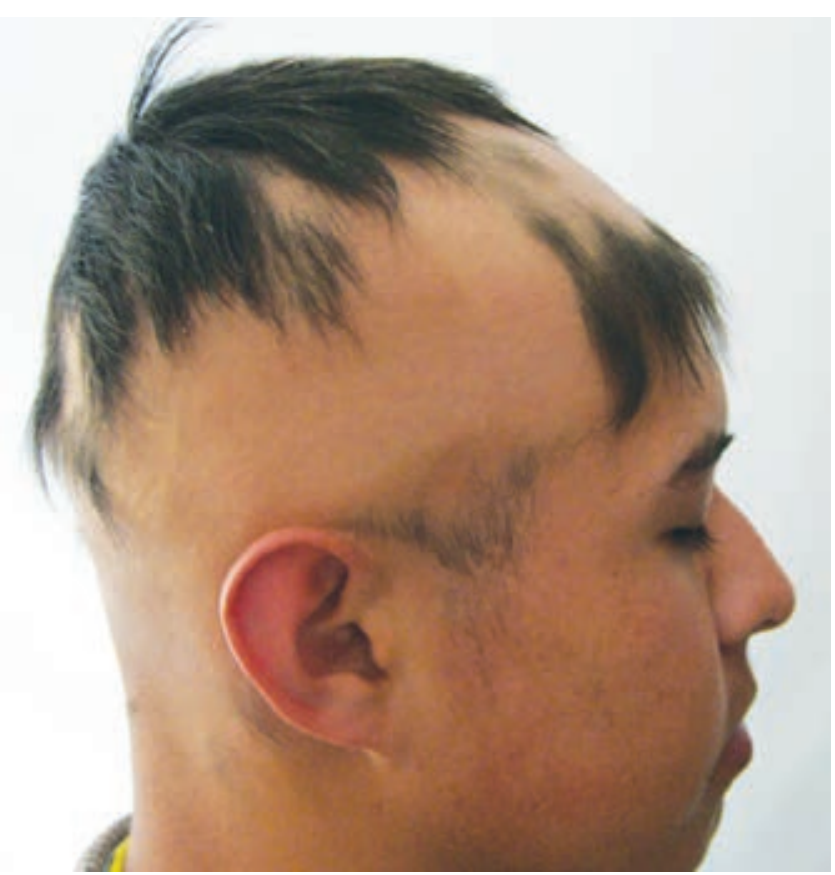

Figura 9: Placas alopécicas de diversos tamaños en piel cabelluda.

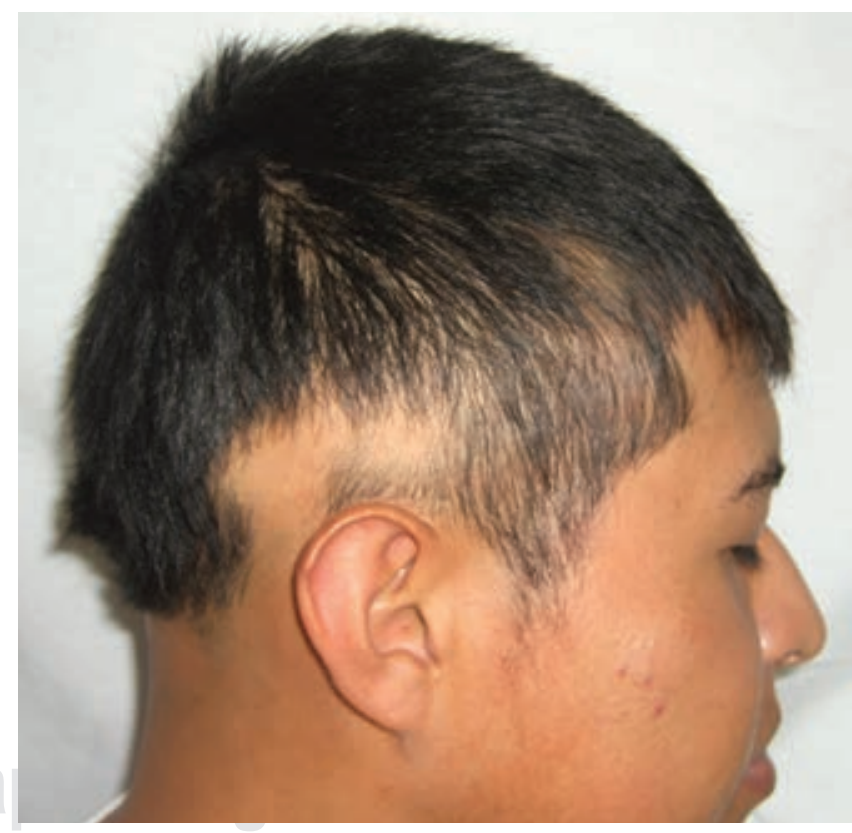

Figura 10: Repoblación de 70\%.

En los pacientes tratados en el Centro Dermatológico «Dr. Ladislao de la Pascua», todos iniciaron con esquemas de primera línea, sin respuesta, por lo que se decidió iniciar con PUVAsol, administrando primero 
8-metoxipsoraleno por vía tópica u oral, con posterior exposición a luz de sol a través de una ventana, con evolución favorable en todos los casos.

Por lo tanto, basados en estos resultados, podemos concluir que esta variante de tratamiento para la $A A$ en placas es una opción prometedora. Sin embargo, se requieren estudios que incluyan más pacientes para valorar el efecto benéfico de la misma.

\section{REFERENCIAS}

1. Villasante-Fricke A, Miteva M. Epidemiology and burden of alopecia areata: a systematic review. Clin Cosmet Investig Dermatol. 2015; 8: 397-403.

2. Martínez-Suárez H. Alopecia areata. Dermatol Rev Mex. 2015; 59: 395-405.

3. Lundin M, Chawa S. Gender differences in alopecia areata. J Drugs Dermatol. 2014; 13: 409-413.

4. Registro de Consulta Externa. Expediente Clínico Electrónico. Centro Dermatológico "Dr. Ladislao de la Pascua". Área de informática y estadística. Enero-Diciembre 2015.

5. Saceda-Corralo D, Grimalt $R$, Fernández-Crehuet $P$, Clemente $A$, Bernárdez C, García-Hernandez MJ et al. Beard alopecia areata: a multicenter review of 55 patients. J Eur Acad Dermatol Venereol. 2017; 31: 187-192.
6. Alkhalifah A, Alsantali A, Wang E, McElwee KJ, Shapiro J. Alopecia areata update. Part II. Treatment. J Am Acad Dermatol. 2010; 62: 191-202.

7. Majumdar B, De A, Ghosh S, Sil A, Sarda A, Lahiri K, Chatterjee G, Das S. "Turban PUVAsol": a simple, novel, effective, and safe treatment option for advanced and refractory cases of alopecia areata. Int $J$ Trichol. 2018; 10: 124-128.

8. Herz-Ruelas M, Gómez-Flores M, Miranda-Maldonado I, Welsh E, Ocampo-Candiani J, Welsh O. Escalating dosimetry of UVA-1 in the treatment of alopecia areata. Int J Dermatol. 2017; 56: 653-659.

9. El-Mofty M, Rasheed H, El-Eishy N, Hegazy RA, Hafez V, ElSamanoudy SI, Shaker O. A Clinical and immunological study of phototoxic regimen of ultraviolet $A$ for treatment of alopecia areata: a randomized controlled clinical trial. J Dermatolog Treat. 2019; 30: 582-587.

10. Welsh O. Phototherapy for alopecia areata. Clin Dermatol. 2016; 34 : 628-632.

Correspondencia:

Dra. María Antonieta Domínguez-Gómez

Dr. Vértiz 464 Esq. Eje 3 Sur,

Col. Buenos Aires, 06780,

Alcaldía Cuauhtémoc, CDMX.

Tel: 55 5519-6351.

E-mail: dominguez035@yahoo.com.mx 